\title{
Curve Matching and Stereo Calibration
}

\author{
John Porrill \& Stephen Pollard
}

The topological obstacles to the matching of smooth curves in stereo images are shown to occur at epipolar tangencies. Such points are good matching primitives, even when the image curves correspond to smooth surface profiles. An iterative scheme for improving camera calibration based on these results is derived and performance demonstrated on real data.

\section{Matching Sets of Points: the 8-Point Algorithm}

We begin by quickly deriving the 8-point algorithm [1] as an introduction to some notation and terminology.

Suppose left and right camera coordinate frames are related by a rotation $R$ and a translation $\mathbf{t}$

$$
\mathbf{p}_{R}=R \mathbf{p}_{L}+\mathbf{t} .
$$

Taking the vector product with $\mathbf{t}$ then the scalar product with $\mathbf{p}_{R}$ gives

$$
\mathbf{p}_{R} \cdot\left(\mathbf{t} \times R \mathbf{p}_{L}\right)=0 .
$$

Introducing the matrix

$$
T=\mathbf{t}^{*}=\left(\begin{array}{ccc}
0 & -t_{z} & t_{y} \\
t_{z} & 0 & -t_{x} \\
-t_{y} & t_{x} & 0
\end{array}\right)
$$

we can write this as

$$
\mathbf{p}_{R}^{t} E \mathbf{p}_{L}=0
$$

where we have defined $E=T R$. Since this equation is homogeneous in $\mathbf{p}_{L}$ and $\mathbf{p}_{R}$ it applies just as well to their projections into the two images

$$
\mathbf{p}_{L} \rightarrow \frac{1}{z_{L}} \mathbf{p}_{L}=\left(\begin{array}{c}
x_{L} \\
y_{L} \\
1
\end{array}\right) \quad \mathbf{p}_{R} \rightarrow \frac{1}{z_{R}} \mathbf{p}_{R}=\left(\begin{array}{c}
x_{R} \\
y_{R} \\
1
\end{array}\right)
$$

Introducing the vector representation of $E$

$$
\mathrm{e}=\left(e_{x x}, e_{x y}, e_{x z}, \ldots, e_{z z}, 1\right)
$$

and the outer product vector

$$
\mathbf{q}=\mathbf{p}_{R} \otimes \mathbf{p}_{L}=\left(x_{R} x_{L}, x_{R} y_{L}, x_{R}, \ldots, 1\right)
$$

${ }^{1}$ AI Vision Research Unit, University of Sheffield, Sheffield S10 2TN.

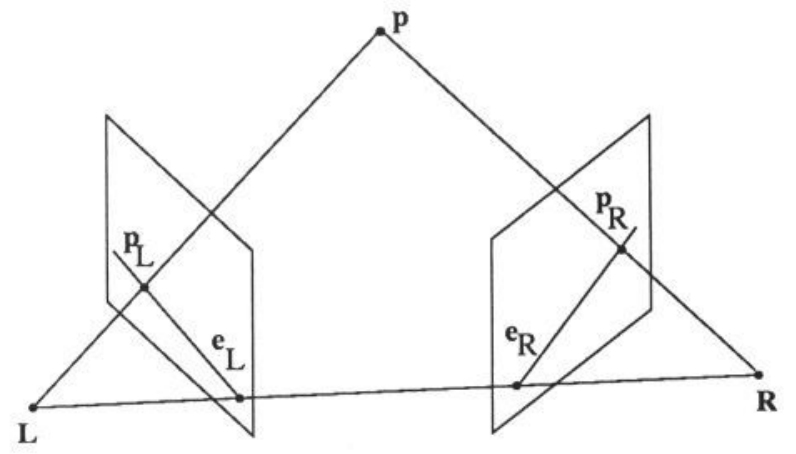

Figure 1: Epipolar Geometry

we can write this as

$$
\mathbf{q}^{t} \mathbf{e}=0
$$

Every matched pair thus gives us a row $\mathbf{q}^{t}$ of the matrix $Q$ in a linear equation

$$
Q \mathrm{e}=0
$$

for e. If we are given at least eight point matches and the matrix $Q$ has $\operatorname{rank}(Q)=8$ we can uniquely recover $E$ up to a scale factor. Conditions on the matched points for the rank condition to be satisfied are given in $[1]$.

Given a point $\mathbf{p}_{L}$ in the left image the corresponding point in the right image must satisfy

$$
\mathbf{m}^{t} \mathbf{p}_{R}=0
$$

where

$$
\mathbf{m}=E \mathbf{p}_{L}
$$

which is the equation of a straight line in the left image. Similarly the point corresponding to $\mathbf{p}_{R}$ in the right image lies on a line $\mathbf{l}^{t} \mathbf{p}_{L}=0$ where $\mathbf{l}=E^{t} \mathbf{p}_{R}$.

These lines are the intersections of the plane through the point $\mathbf{p}$ in the world and the centres of the left and right cameras with the two camera focal planes (see Figure 1). These epipolar lines are formed by the intersections of the pencil of planes through the line joining the image centres with the camera focal planes. Hence such lines form a pencil passing through the point of intersection of the line of centres with the focal plane, we will call this point the epipolar base point. The condition that all possible lines $\mathbf{m}=E \mathbf{p}_{L}$ form a pencil is satisfied only if $\operatorname{rank}(E) \leq 2$, but this follows from the non-linear constraint on $E$ that $\operatorname{det}(E)=$ $\operatorname{det}(T) \operatorname{det}(R)=0$. 

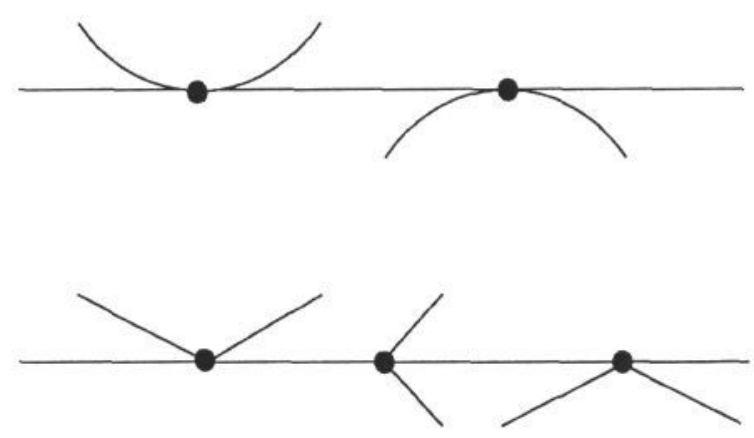

Figure 2: The 5 Curve-Epipolar Singularities

There are two other non-linear constraints that have to be imposed to ensure that $E$ is of the form $T R$. They can easily be derived from the equation $E E^{T}=-T^{2}$. The conventional procedure for solving these equations is to find a least squares solution of equation 2 and then a least squares solution of the constraints, however this procedure can produce biased estimates. An alternative is to enforce the constraints while minimising image plane errors, this is the procedure we adopt $[2,3]$.

\section{Curve Matching}

When the epipolars are known it is easy to determine whether two curves can be matched. The intersections with corresponding epipolars must have the same numbers of points. In principle this imposes an infinite number of conditions, one for each epipolar. However we do not need to check every epipolar-for generic curves we need only check that the epipolar tangencies agree in number and type. Agreement for the portions between is then guaranteed. Since the epipolars are ordered there are two types of tangency, convex and concave (inflexion tangencies are not generic). If we are dealing with piecewise smooth curves we must also check that corners match. There are three types of corner. These generic curve-epipolar singularities are shown in Figure 2. It is obvious that corresponding epipolar tangencies are good matching primitives, i.e. they satisfy equation 1 . Of course, they are not immediately useful for calculating $E$ since we must know the epipolars before the tangencies can be located.

If the epipolars are unknown we can eliminate the possibility of a match by showing that the epipolar singularities cannot correspond for any choice of epipolar base points. This can be done systematically by dividing space into an aspect graph of regions in which the curves have stable descriptions. As the epipolar base point moves the type and number of epipolar tangen-
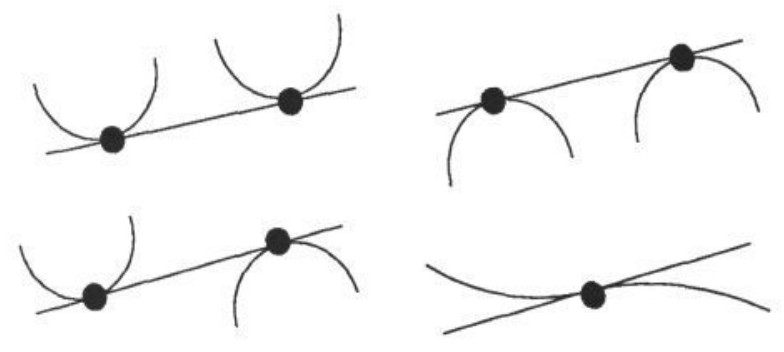

Figure 3: The Stable Region Boundaries

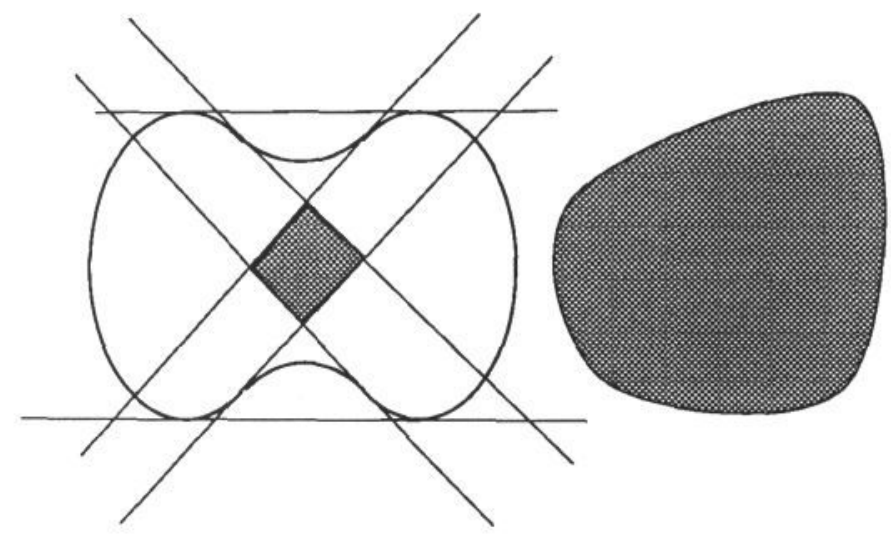

Figure 4: Matchable Curves for Epipolars Based in the Shaded Region

cies does not change until a region boundary is crossed, then there is a catastrophic change in the description. The stable boundaries between such regions are enumerated in Figure 3 for the case of smooth curves. Basically they consist of the curve itself, inflexion lines and bi-tangents for smooth curves; lines joining corners to corners or to tangencies are also region boundaries for piecewise smooth curves.

If the descriptions in the left and right images are different for every such region (of which there are only a finite number), then the two curves cannot be matched. If they are the same for some region then matching may be possible, but the epipolars on which corresponding singularities lie must be in projective correspondence. Sometimes checking this is trivial. For example the curves of Figure 4 have trivial descriptions for epipolar base points in the regions shown, and the possibility of matching is immediate.

If this is not the case we have a set of singularities $\mathbf{p}_{L}^{i}(E), \mathbf{p}_{R}^{i}(E) i=1, \ldots, N$ smoothly dependent on the epipolar geometry $E$ and we must find a value of $E$ for which

$$
f^{i}(E)=\mathbf{p}_{R}^{i}(E)^{t} E \mathbf{p}_{L}^{i}(E)=0 .
$$

In general this equation must be solved by search or 
optimisation methods.

When two curves have corresponding epipolar tangencies at $\mathbf{p}_{L}, \mathbf{p}_{R}$ the tangent vectors at the tangencies $\mathbf{t}_{L}$ must lie along the epipolar lines. At tangencies we thus have two extra linear conditions on $E$

$$
\mathbf{t}_{R}^{t} E \mathbf{p}_{L}=\mathbf{p}_{R}^{t} E \mathbf{t}_{L}=0
$$

\section{Uniqueness of Solutions}

Due to the non-linearity of equation 3 it is rather too much to hope to prove uniqueness of solutions. However we can in some cases prove that the solutions are isolated.

Suppose we have 8 matching primitives and a solution $E_{0}$ of equation 3. Choose a non-zero element of $E_{0}$ and fix its value so that the scale factor for nearby matrices $E$ is fixed. Then we have a map $F: R^{8} \rightarrow R^{8}$

$$
F: E \rightarrow\left(f^{1}(E), \ldots, f^{8}(E)\right)^{t}: E_{0} \rightarrow 0
$$

As $E$ changes the points $\mathbf{p}(E)$ move along their respective curves so that

$$
\frac{\partial \mathbf{p}}{\partial E}=\frac{\partial s}{\partial E} \mathbf{t}
$$

where $s$ is arc-length. Hence we can calculate the derivatives of the $f^{i}$ to be

$$
\frac{\partial f}{\partial E}=\mathbf{p}_{R} \otimes \mathbf{p}_{L}+\frac{\partial s_{R}}{\partial E} \mathbf{t}_{R}^{t} E \mathbf{p}_{L}+\frac{\partial s_{L}}{\partial E} \mathbf{p}_{R}^{t} E \mathbf{t}_{L}
$$

Due to the epipolar tangency the second two terms are always zero at $E=E_{0}$ so that

$$
\frac{\partial F}{\partial E}\left(E_{0}\right)=Q
$$

the matrix of outer products for the matched points. Hence the non-linear equations have exactly the same degeneracy as those of the linear equations in some neighbourhood of the known solution. In particular if the matches satisfy the conditions for $Q$ to have rank 8 the solution $E_{0}$ is isolated (by the inverse function theorem [6]).

\section{Newton-Raphson Iteration}

Suppose $E_{0}$ is an approximation to the epipolar geometry. In light of the last section it is sensible to try to improve $E$ by the Newton-Raphson method, that is, we solve $F(E)=0$ by approximating

$$
F(E) \approx F\left(E_{0}\right)+\frac{\partial F}{\partial E}\left(E_{0}\right) \cdot\left(E-E_{0}\right)
$$

and solving this linear equation for $E-E_{0}$.

This becomes much clearer if we think in terms of small changes to $\mathbf{p}_{L}, \mathbf{p}_{R}, E_{0}$ chosen to make

$$
\left(\mathbf{p}_{R}+\delta \mathbf{p}_{R}\right)^{t}\left(E_{0}+\delta E\right)\left(\mathbf{p}_{L}+\delta \mathbf{p}_{L}\right)=0 .
$$

since these changes must be made along the curves, and removing second order quantities, we have

$$
\mathbf{p}_{R}^{t}\left(E_{0}+\delta E\right) \mathbf{p}_{L}+\delta s_{R} \mathbf{t}_{R} E_{0} \mathbf{p}_{L}+\delta s_{L} \mathbf{p}_{R}^{t} E_{0} \mathbf{t}_{L}=0 .
$$

The second two terms are second order small by equation 4 and so the corrected $E$ satisfies $\mathbf{p}_{R}^{t} E \mathbf{p}_{L}=0$ to linear order. That is, in calculating $E$ we can use approximate epipolar tangencies in equation 1 as if they were true matching points.

Since this is a Newton-Raphson approximation, in a neighbourhood of the exact solution the procedure is quadratically convergent to the true solution. If we wish to iterate we need to find the new epipolar tangencies for the corrected $E$-matrix. The tangencies will move by small amounts $\delta s_{L}, \delta s_{R}$ along the curves, and must satisfy the second two parts of equation 3 . From $\mathbf{p}_{R}^{t} E \mathbf{t}_{L}=0$ an $\delta \mathbf{t}_{L}=\delta s_{L} \kappa_{L} \mathbf{n}_{L}$ where $\kappa_{L}$ is the curvature we get

$$
\left(\mathbf{p}_{R}+\delta s_{R} \mathbf{t}_{R}\right)^{t} E\left(\mathbf{t}_{L}+\delta s_{L} \kappa_{L} \mathbf{n}_{L}\right)=0
$$

ignoring second order quantities we have

$$
\mathbf{p}_{R}^{t} E \mathbf{t}_{L}+\delta s_{R} \mathbf{t}_{R}^{t} E \mathbf{t}_{L}+\delta s_{L} \kappa_{L} \mathbf{p}_{R}^{t} E \mathbf{n}_{L}=0
$$

and from $\mathbf{t}_{R}^{t} E \mathbf{p}_{L}=0$ we get

$$
\mathbf{t}_{R}^{t} E \mathbf{p}_{L}+\delta s_{R} \kappa_{R} \mathbf{n}_{R}^{t} E \mathbf{p}_{L}+\delta s_{L} \mathbf{t}_{L}^{t} E \mathbf{p}_{L}=0
$$

giving a pair of linear equations for the changes in position along the curve.

\section{Positional Accuracy}

The data from the epipolar tangencies can be combined with other matching data from high curvature edge points or corner detector output to obtain optimal calibration accuracy. To facilitate this we need an estimate of the positional accuracy of these primitives. If the angular error in the epipolars is $\theta$ then the perpendicular error from the epipolar is

$$
\epsilon=2 \rho \tan ^{2} \frac{\theta}{2} \approx \frac{1}{2} \rho \theta^{2}
$$




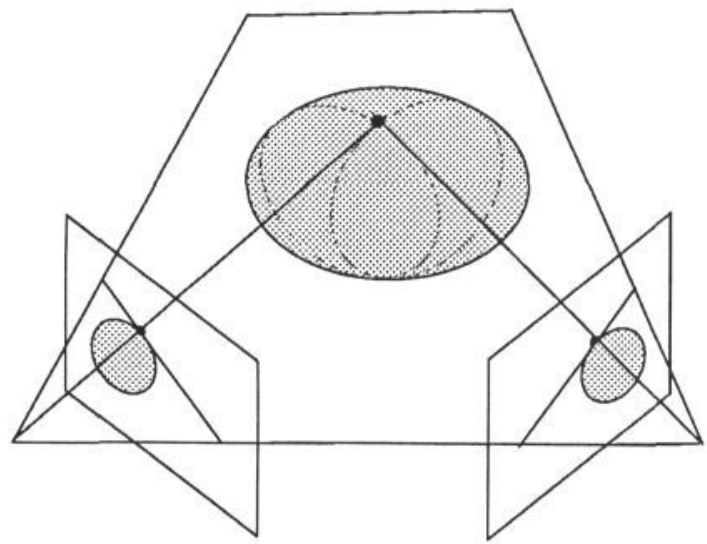

Figure 5: An Epipolar Tangency for a Profile Curve is a Real Point

and so is quadratic in the error in the epipolars, as we already knew, and linear in the radius of curvature. Matches are thus most accurate for very sharp tangencies, that is, corners. However tangencies which are very far from being corners are still useful for matching. For example with initial epipolars in error by about $5^{\circ} \approx 0.1$ radian a matching accuracy of 0.5 pixels can be obtained if

$$
\frac{1}{2} \rho \theta^{2}=0.005 \rho<0.5
$$

that is for

$$
\rho<100 \text { pixel. }
$$

In fact due to the extended nature of edges, for radii of curvature up to 20 pixels they can probably be located more accurately than, say, corner detector output.

\section{Profile Curves}

It is interesting to note that even when the edges seen in the image correspond to the profiles of smooth objects in the world, and so do not correspond to any single real curve in the world, the epipolar tangencies do correspond to real points: the points where the epipolar planes are tangent to the smooth objects, see Figure 5. This observation has been used in [5] to identify the rotation axis of an object from two orthographic projections. Its importance to us is that all the main types of intensity edges can produce useful matching primitives.

\section{$7 \quad$ Results}

Figure 6 shows a real scene viewed with a convergent camera geometry. We take as starting approximation a parallel camera geometry

$$
E=\left(\begin{array}{rrr}
0 & 0 & 0 \\
0 & 0 & -1 \\
0 & 1 & 0
\end{array}\right)
$$

for which the epipolar lines are corresponding rasters. Figure 7 shows the stereo disparities obtained using the PMF stereo algorithm [4] with this incorrect geometry. Though no incorrect curves are matched, the geometry is grossly distorted.

After edge detection some corresponding epipolar tangencies are located and matched between the images. The results are shown in Figure 8. Using these matches a new epipolar geometry is calculated. Figure 9 shows the new epipolars superimposed on the edge map. I1 can be seen that a convergent geometry has been recovered. When this viewing geometry is used by PMF the much improved disparity maps shown in Figure 10 result.

\section{Acknowledgements}

We would like to thank Neil Thacker for useful discussions on calibration from image data and the use of his calibration software. This research was supported by SERC project grant no. GR/E 49616 awarded under the ACME programme in collaboration with the IBM Scientific Centre, Winchester.

\section{References}

[1] Longuet-Higgins, H. C. "A computer algorithm for reconstructing a scene from two projections" Nature Vol. 293 (1981) pp 133-135.

[2] Thacker, N. A. and Mayhew J. E. W. "Stereo camera calibration from arbitrary images" AIVRU Memo No. 041 (1989).

[3] Trivedi, H. P. "Estimation of stereo and motion parameters using a variational principle" Image and Vision Computing Vol. 5 (1987) pp 181-183.

[4] Pollard, S. B., Mayew J. E. W. and Frisby, J. P. "PMF: A stereo correspondence algorithm using a disparity gradient limit" Perception Vol. 14 (1985) pp 449-470.

[5] Rieger J. H. "Three-dimensional motion from fixed points of a deforming profile curve" Optics Letters Vol. 11 (1986) pp 123-125.

[6] Rudin, W. "Principles of mathematical analysis" McGraw Hill, New York (1964). 


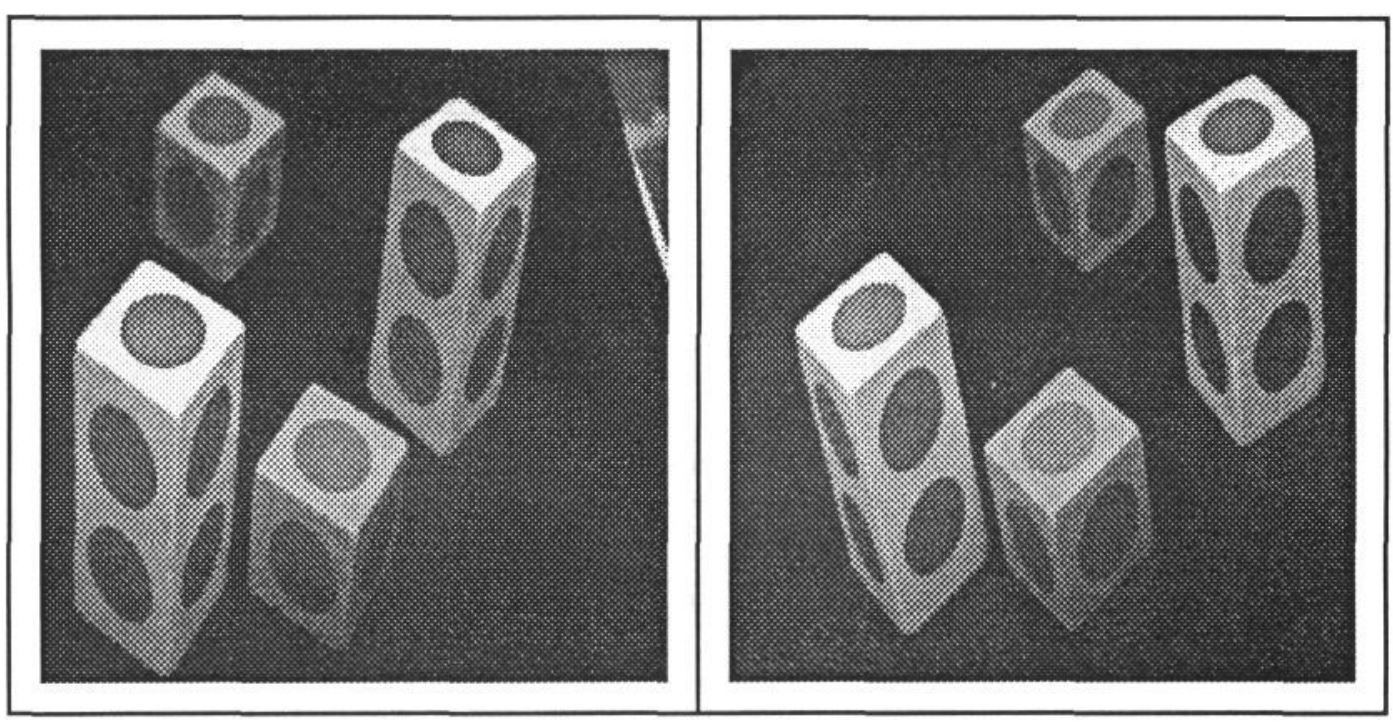

Figure 6: Stereo Image of Toy Blocks
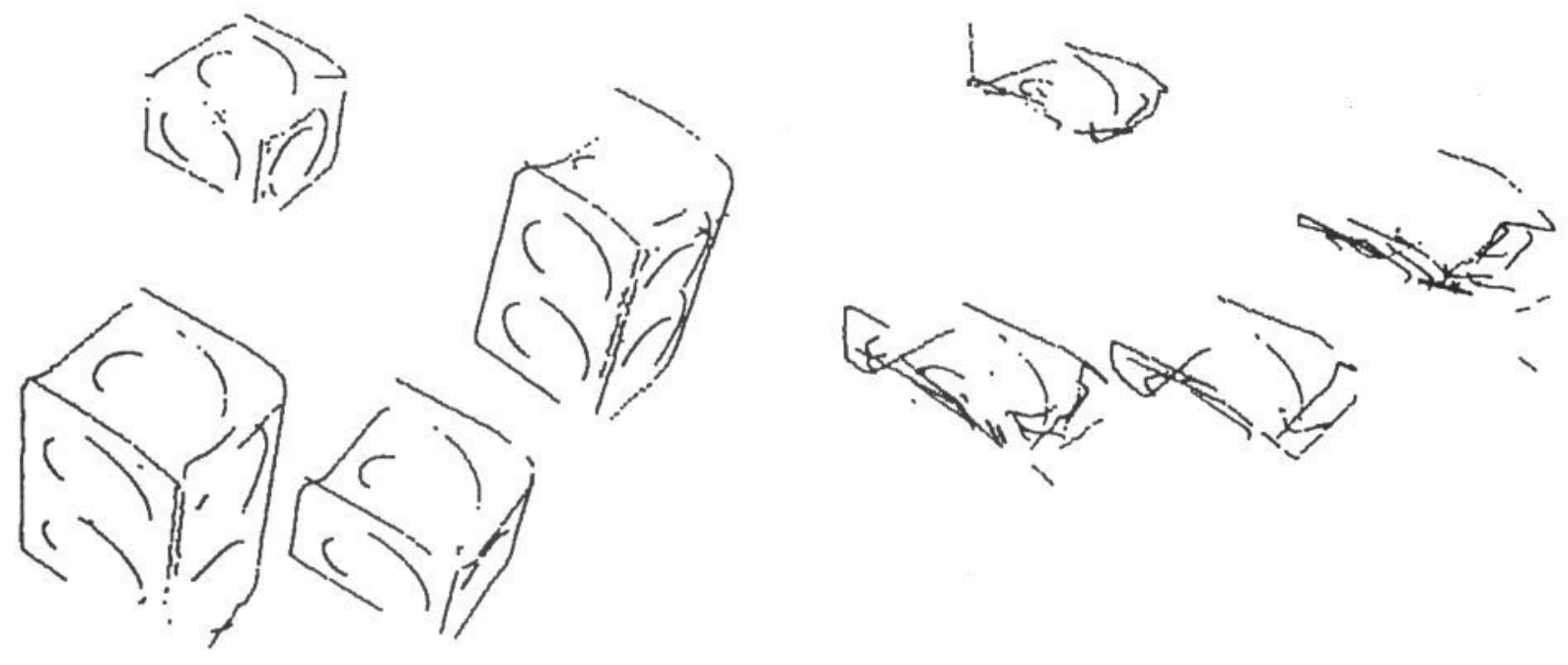

Figure 7: PMF Stereo Correspondences Using Incorrect Camera Geometry
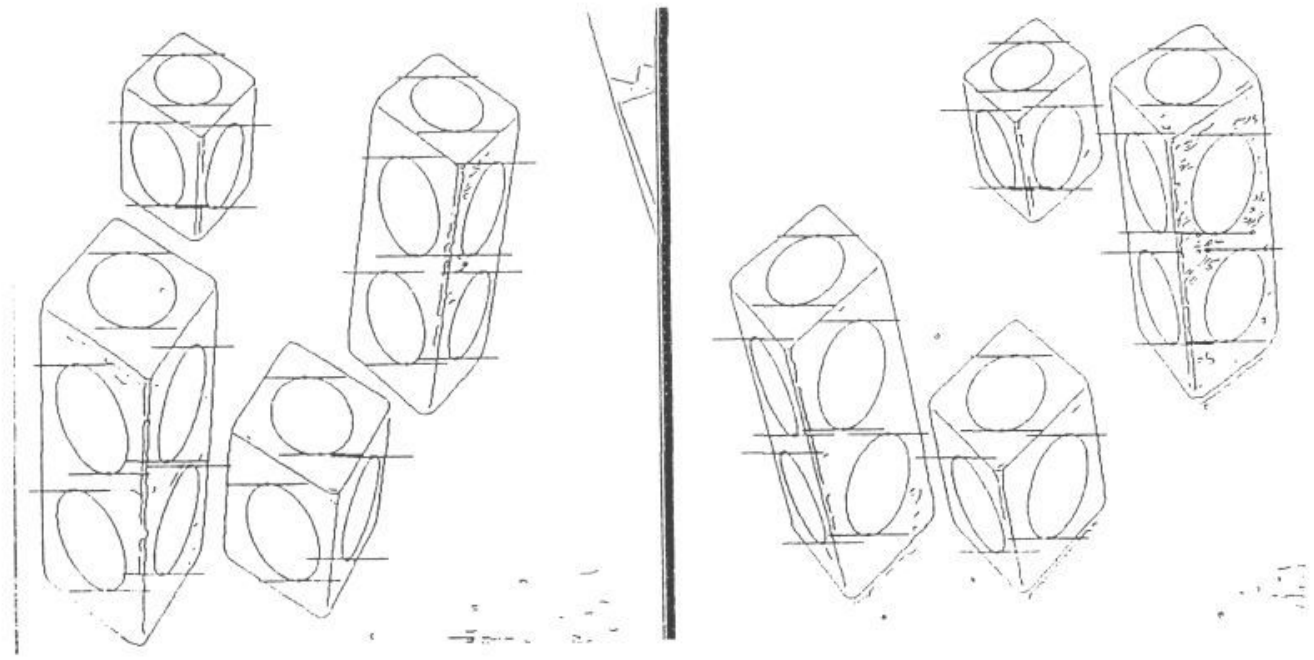

Figure 8: Some Matching Epipolar Tangencies 


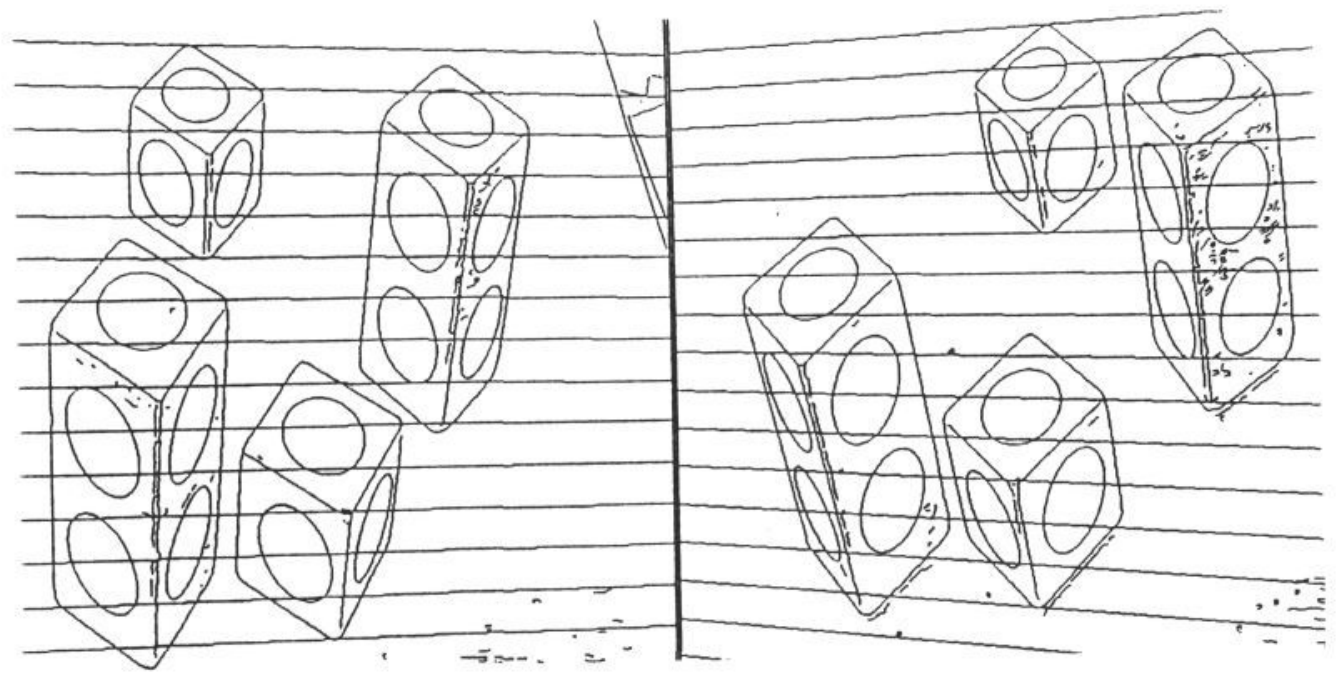

Figure 9: Epipolar Grid and Some Corresponding Epipolars
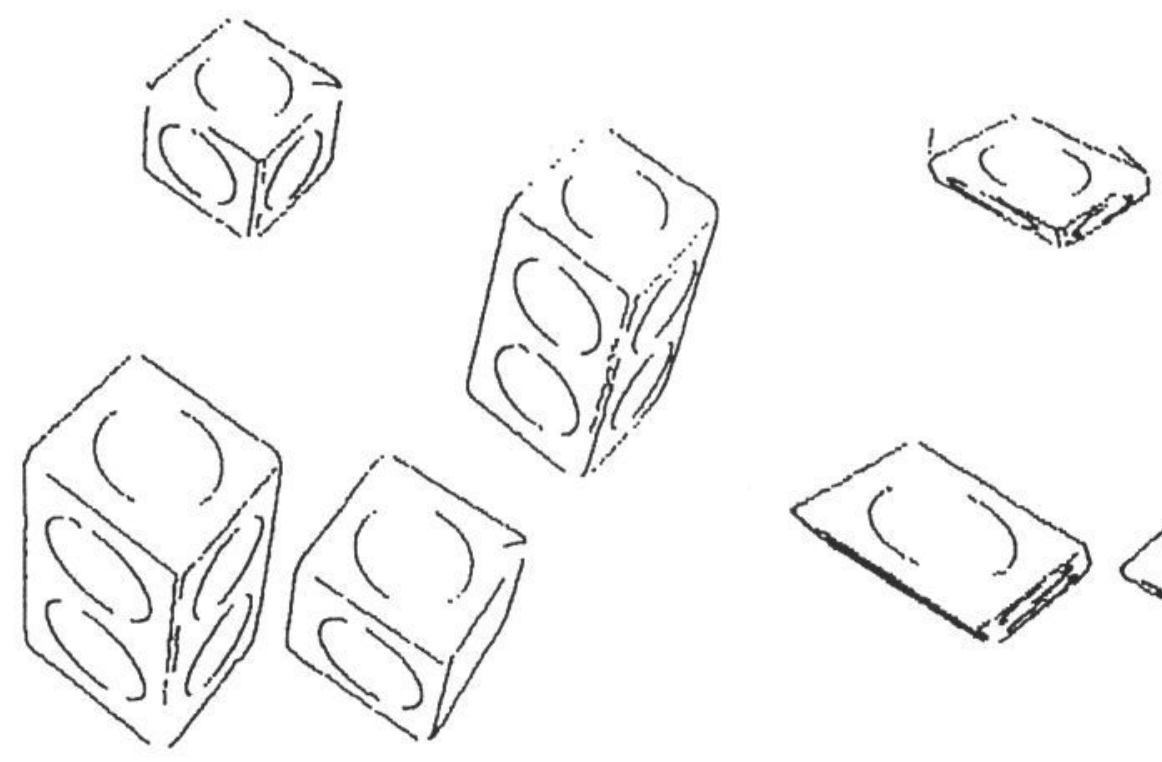

Figure 10: PMF Stereo Correspondences Using New Camera Geometry 